\title{
Cell Active Hydroxylactam Inhibitors of Human Lactate Dehydrogenase with Oral Bioavailability in Mice
}

Hans E. Purkey ${ }^{\mathrm{a}^{\mathrm{a}}}$, Kirk Robarge ${ }^{\mathrm{a}}$, Jinhua Chen ${ }^{\mathrm{b}}$, Zhongguo Chen ${ }^{\mathrm{b}}$, Laura B. Corson ${ }^{\mathrm{a}}$, Charles Z. Ding ${ }^{\mathrm{b}}$, Antonio DiPasquale ${ }^{c}$, Peter S. Dragovich ${ }^{a}$, Charles Eigenbrot ${ }^{a}$, Marie Evangelista ${ }^{a}$, Benjamin P. Fauber ${ }^{a}$, Zhenting Gao ${ }^{b}$, Hongxiu Ge ${ }^{b}$, Anna Hitz ${ }^{a}$, Qunh Ho ${ }^{b}$, Sharada S. Labadie ${ }^{a}$, Kwong Wah Lai ${ }^{b}$, Wenfeng $\mathrm{Liu}^{\mathrm{b}}$, Yajing Liu ${ }^{\mathrm{b}}$, Chiho Lib ${ }^{\mathrm{b}}$, Shuguang Ma ${ }^{\mathrm{a}}$, Shiva Malek ${ }^{\mathrm{a}}$, Thomas O’Brien ${ }^{\mathrm{a}}$, Jodie Pang ${ }^{\mathrm{a}}$, David Peterson $^{\mathrm{a}}$, Laurent Salphati ${ }^{\mathrm{a}}$, Steven Sideris ${ }^{\mathrm{a}}$, Mark Ultsch $^{\mathrm{a}}$, BinQing Wei ${ }^{\mathrm{a}}$, Ivana Yen ${ }^{\mathrm{a}}$, Qin Yue ${ }^{\mathrm{a}}$, Huihui Zhang $^{\mathrm{b}}$, Aihe Zhou ${ }^{\mathrm{a}}$

${ }^{a}$ Genentech, Inc., 1 DNA Way, South San Francisco, California 9408o, United States

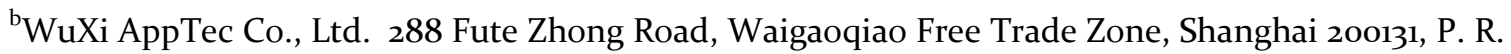
China

${ }^{c}$ College of Chemistry, University of California, Berkeley, Berkeley, California, 94720, United States

\section{Supporting Information}

In vitro drug treatment experiments. All cell lines were obtained from our in-house tissue culture cell bank (original source was ATCC). Lines were authenticated by short tandem repeat (STR) and genotyped upon re-expansion. Cells were maintained in RPMI 1640 media supplemented with 10\% FBS (Sigma; F2442). Cells were plated using optimal seeding densities in 384-well plates using RPMI, 5\% FBS (Sigma F4135), $100 \mathrm{ug} / \mathrm{ml}$ penicillin, 100 units/ml streptomycin (Gibco 15140-122). Optimal seeding densities were established for each cell line in order to reach $75-80 \%$ confluence at the end of the assay. The following day, cells were treated with compound 29 using a 6 pt dose titration scheme. After 72 hours, cell viability was assessed using the CellTiter-Glo ${ }^{\circledR}$ Luminescence Cell Viability assay. Absolute inhibitory concentration (IC) values were calculated using four-parameter logistic curve fitting. 


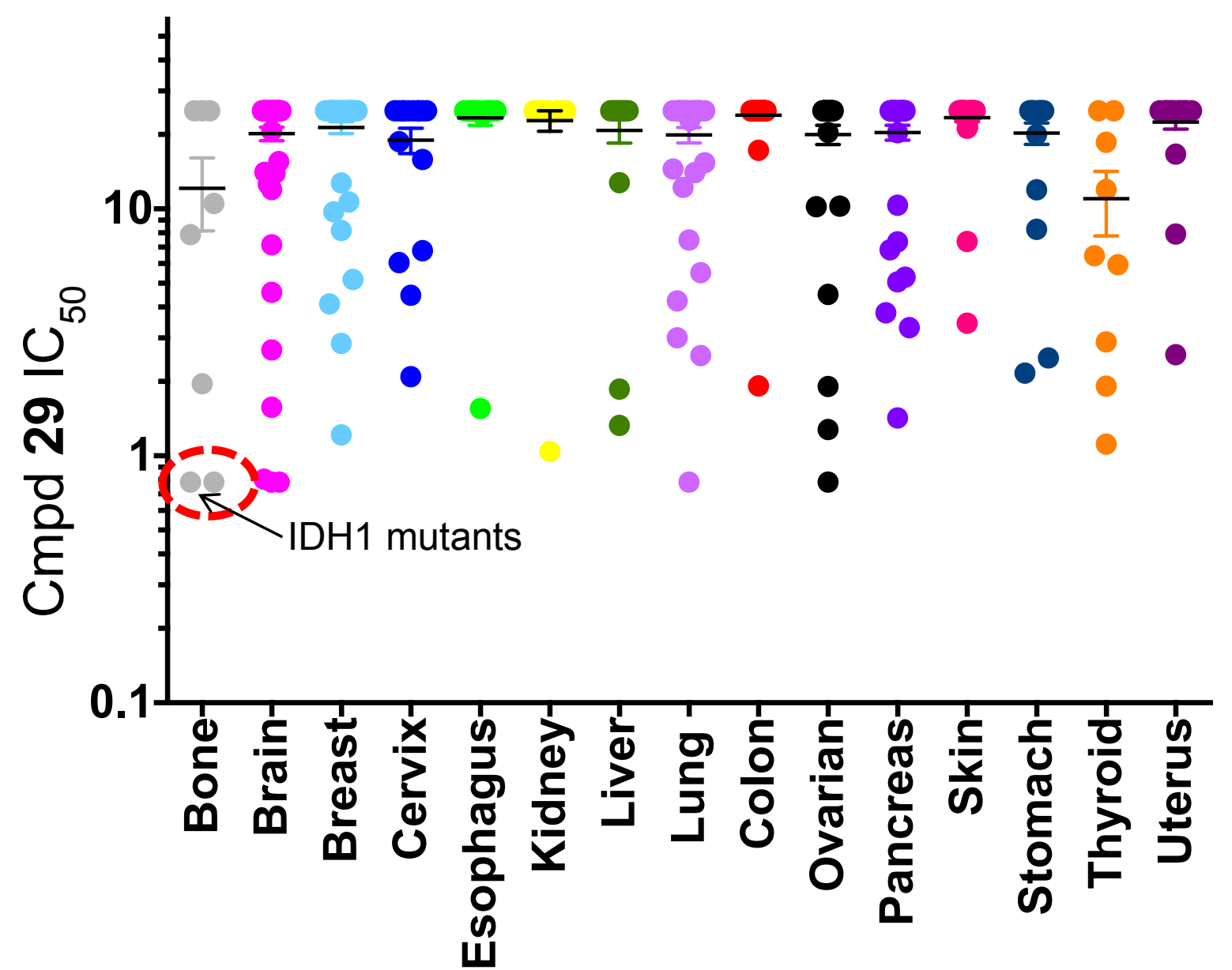

Figure S1. Inhibition of cell proliferation by 29 in 347 cancer cell lines. Cells were grown in the presence of $\mathbf{2 9}$ for 72 hours.

Table S1. Proliferation IC50s for 29 in 37 sensitive cancer cell lines

$\begin{array}{llrr}\text { Cell Line } & \text { Tissue } & \text { CELLINE_ID } & \text { IC50 } \\ \text { 105KC } & \text { Bone } & 586052 & 0.78 \\ \text { JJ012 } & \text { Bone } & 586055 & 0.78 \\ \text { 143B } & \text { Bone } & 584976 & 2.0 \\ \text { G84 } & \text { Brain } & 132488 & 0.78 \\ \text { 1321N1 } & \text { Brain } & 586793 & 0.78 \\ \text { ONS-76 } & \text { Brain } & 586962 & 0.80 \\ \text { G22 } & \text { Brain } & 132482 & 1.6 \\ \text { G140 } & \text { Brain } & 132425 & 2.7 \\ \text { G96 } & \text { Brain } & 132516 & 4.6 \\ \text { HCC1143 } & \text { Breast } & 585292 & 1.2 \\ \text { CAL-120 } & \text { Breast } & 130224 & 2.9 \\ \text { Hs 578T } & \text { Breast } & 130032 & 4.1 \\ \text { SISO } & \text { Cervix } & 586885 & 2.1 \\ \text { SKG-II } & \text { Cervix } & 586968 & 4.5 \\ \text { HCT-15 } & \text { Colon } & 132492 & 1.9 \\ \text { KYSE-520 } & \text { Esophagus } & 586703 & 1.6\end{array}$




$\begin{array}{lllr}\text { KMRC-1 } & \text { Kidney } & 586966 & 1.0 \\ \text { SNU-475 } & \text { Liver } & 586253 & 1.3 \\ \text { SNU-423 } & \text { Liver } & 586251 & 1.9 \\ \text { HT-1080 } & \text { Lung } & 585278 & 0.78 \\ \text { NCl-H1437 } & \text { Lung } & 129264 & 2.5 \\ \text { NCl-H1339 } & \text { Lung } & 586367 & 3.0 \\ \text { LXF-289 } & \text { Lung } & 586341 & 4.2 \\ \text { MCAS } & \text { Ovary } & 586706 & 0.78 \\ \text { PA-1 } & \text { Ovary } & 586195 & 1.3 \\ \text { Hs 38.T } & \text { Ovary } & 134160 & 1.9 \\ \text { 59M } & \text { Ovary } & 134179 & 4.5 \\ \text { MIA PaCa-2 } & \text { Pancreas } & 584217 & 1.4 \\ \text { SW 1990 } & \text { Pancreas } & 584189 & 3.3 \\ \text { PSN1 } & \text { Pancreas } & 584456 & 3.8 \\ \text { GR-M } & \text { Skin } & 586575 & 3.4 \\ \text { Hs 746T } & \text { Stomach } & 587042 & 2.2 \\ \text { MKN-74 } & \text { Stomach } & 586301 & 2.5 \\ \text { S-117 } & \text { Thyroid } & 586883 & 1.1 \\ \text { FTC-238 } & \text { Thyroid } & 586815 & 1.9 \\ \text { B-CPAP } & \text { Thyroid } & 586852 & 2.9 \\ \text { HEC-265 } & \text { Uterus } & 586958 & 2.6\end{array}$

\section{Mouse Pharmacokinetics Study}

The pharmacokinetics of compound $\mathbf{2 9}$ was evaluated following a single intravenous bolus (IV) dose of $1.0 \mathrm{mg} / \mathrm{kg}$ and oral administration (PO) of solution/amorphous suspension at a dose of $5 \mathrm{mg} / \mathrm{kg}$ in female $\mathrm{CD}-1$ mice $(\mathrm{N}=3)$. The vehicle used for IV administration was 10/50/40 EtOH/PEG400/50mM citrate $\mathrm{pH} 3$ (v/v, 10/50/40), and for PO, 0.5\% methycellulose:0.2\% Tween in water (MCT). Blood samples for the IV dose group were collected at $0.033,0.25,1,2,4,6$ hours post dose. Blood samples for PO dose groups were collected at $0.25,0.5,1,2,4$, and 6 hours post dose. For the high dose oral PK study at 50, 100 , and $200 \mathrm{mg} / \mathrm{kg}$, blood samples were collected at $0.25,0.5,1,2,4,6$, and 8 hours post dose. Blood samples were centrifuged within 29 minutes of collection, and plasma was harvested. Plasma samples were stored at approximately $-70^{\circ} \mathrm{C}$ until the analysis of the compound concentration by a liquid chromatography/tandem mass spectrometry (LCMS/MS) method. PK parameters were determined by non-compartmental methods using WinNonlin (version 5.2, Pharsight Corporation, Mountain View, CA). 


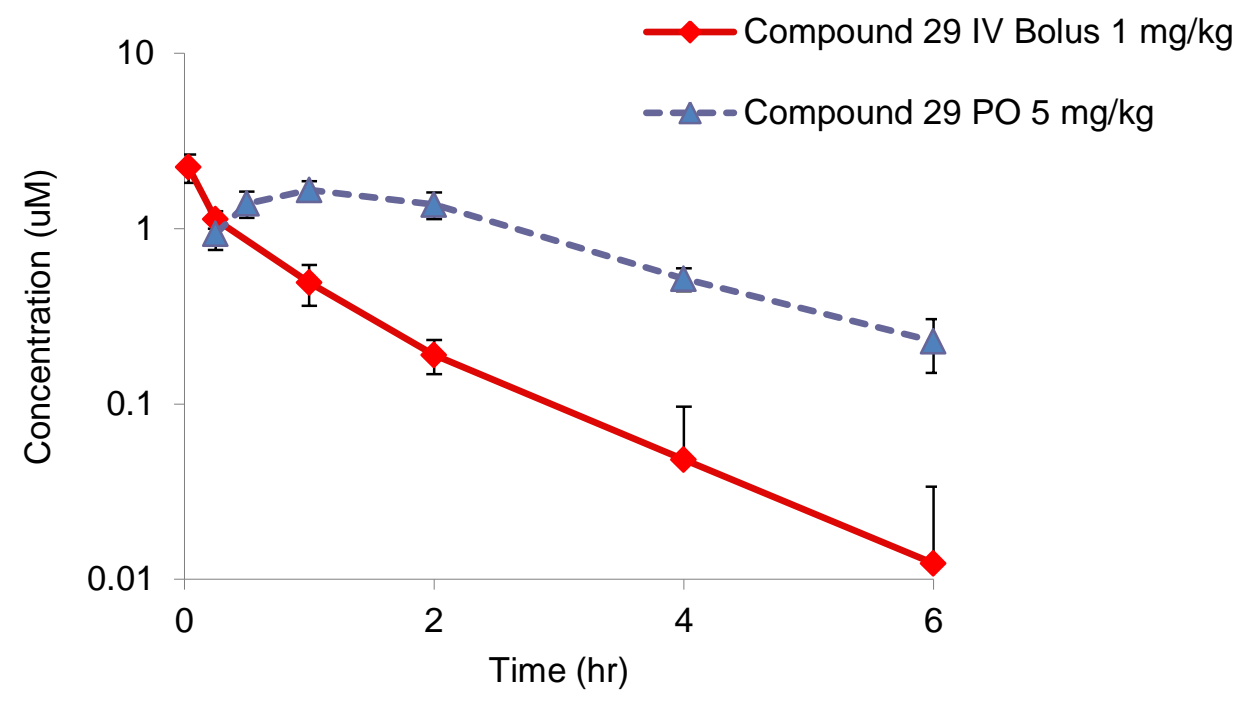

Figure S2. Blood concentration-time profiles of compound 29 in mice following IV (1 $\mathrm{mg} / \mathrm{kg})$ and $\mathrm{PO}(5 \mathrm{mg} / \mathrm{kg})$ administrations.

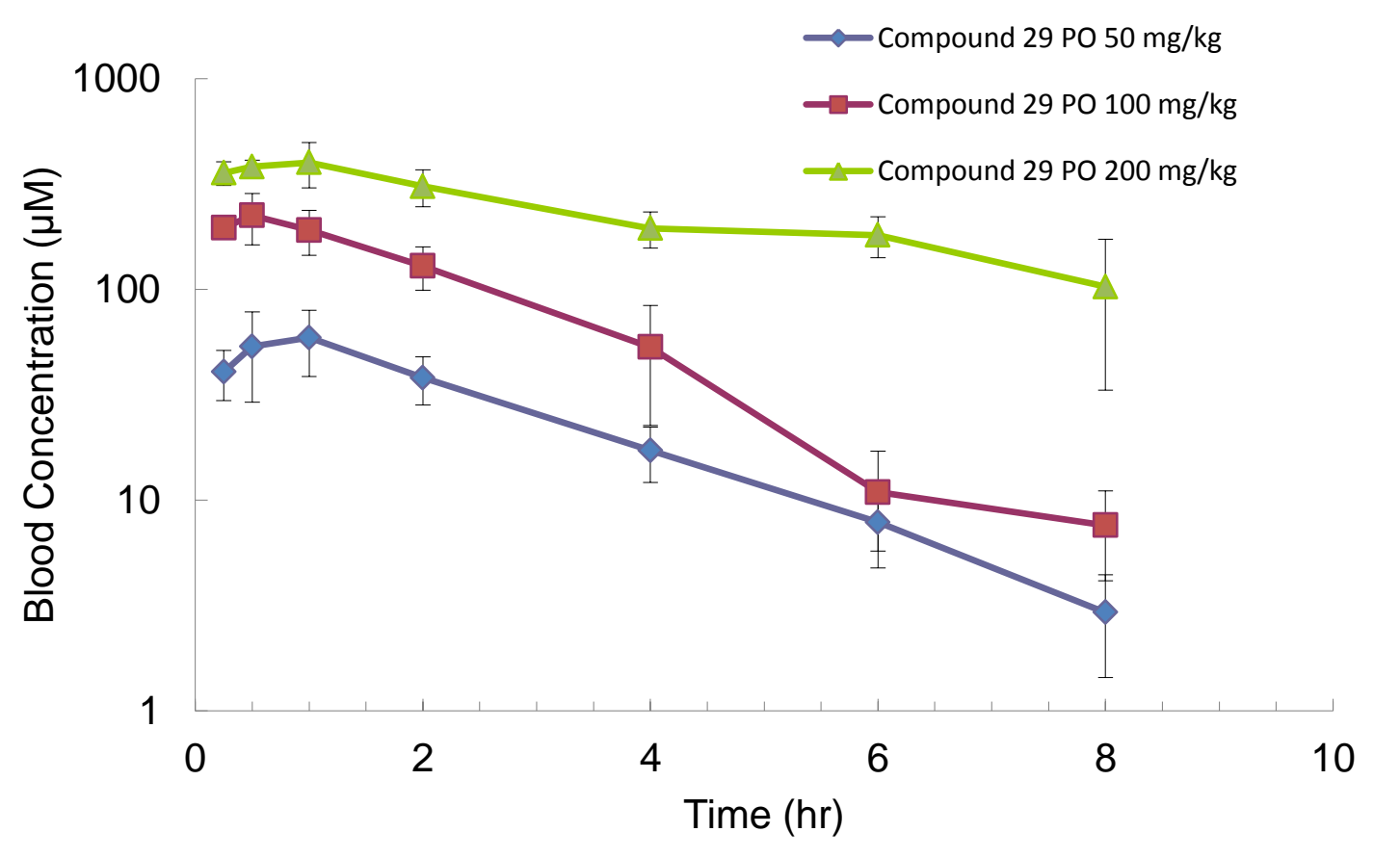

Figure S3. Blood concentration-time profiles of compound 29 in mice following PO $(50,100$ and $200 \mathrm{mg} / \mathrm{kg}$ ) administrations 
Purity and LCMS Analysis of Compounds

\begin{tabular}{|c|c|c|c|}
\hline Compound & MW & LC-MS ESI $^{+}[\mathrm{M}+1]^{+}$ & LC-MS Purity (254 nm) [\%] \\
\hline 1 & 331.04 & 332.1 & 95 \\
\hline 2 & 407.07 & 408.1 & 100 \\
\hline 3 & 414.03 & 415.0 & 98 \\
\hline 4 & 413.03 & 414.1 & 100 \\
\hline 5 & 414.03 & 415.1 & 100 \\
\hline 6 & 431.02 & 431.7 & 99 \\
\hline 7 & 490.94 & 492.0 & 97 \\
\hline 8 & 428.04 & 429.1 & 100 \\
\hline 9 & 429.03 & 430.1 & 96 \\
\hline 10 & 438.03 & 438.9 & 98 \\
\hline 11 & 453.06 & 453.8 & 94 \\
\hline 12 & 443.04 & 444.1 & 96 \\
\hline 13 & 456.07 & 456.8 & 95 \\
\hline 14 & 429.03 & 429.7 & 96 \\
\hline 15 & 498.08 & 499.0 & 100 \\
\hline 16 & 512.10 & 513.2 & 100 \\
\hline 17 & 510.12 & 511.2 & 93 \\
\hline 18 & 522.06 & 523.1 & 100 \\
\hline 19 & 513.08 & 513.9 & 93 \\
\hline 20 & 523.05 & 523.8 & 96 \\
\hline 21 & 495.11 & 495.8 & 99 \\
\hline 22 & 497.10 & 498.2 & 96 \\
\hline 23 & 553.13 & 554.2 & 92 \\
\hline 24 & 526.12 & 526.9 & 98 \\
\hline 25 & 496.10 & 497.1 & 98 \\
\hline 26 & 482.09 & 483.1 & 97 \\
\hline 27 & 539.11 & 539.9 & 99 \\
\hline 28 & 498.08 & 499.1 & 96 \\
\hline 29 & 498.08 & 499.1 & 99 \\
\hline 30 & 498.08 & 499.1 & 96 \\
\hline 31 & 526.12 & 527.0 & 97 \\
\hline 32 & 512.06 & 512.8 & 97 \\
\hline 33 & 526.12 & 526.9 & 98 \\
\hline 34 & 546.05 & 546.8 & 93 \\
\hline 35 & 538.12 & 539.0 & 92 \\
\hline 36 & 521.10 & 522.0 & 95 \\
\hline
\end{tabular}

Experiments performed on an Agilent 1200 UHPLC coupled with Agilent MSD (6140) mass spectrometer using ESI as ionization source. The LC separation was using a Agilent ZORBAX SB-C18, 1.8um, 2.1*50mm column with a $0.4 \mathrm{ml} /$ minute flow rate. Solvent A is water with $0.1 \% \mathrm{FA}$ and solvent $\mathrm{B}$ is acetonitrile with $0.1 \% \mathrm{FA}$. The gradient consisted with $2-98 \%$ solvent B over $7 \mathrm{~min}$ and hold $98 \% \mathrm{~B}$ for $1.5 \mathrm{~min}$ following equilibration for $1.5 \mathrm{~min}$. LC column temperature is $40{ }^{\circ} \mathrm{C}$. UV absorbance was collected at $220 \mathrm{~nm}$ and $254 \mathrm{~nm}$ and mass spec full scan was applied to all experiment. 


\section{Synthetic procedures and characterization data for key compounds tested in cellular assays.}

Synthesis of 46:

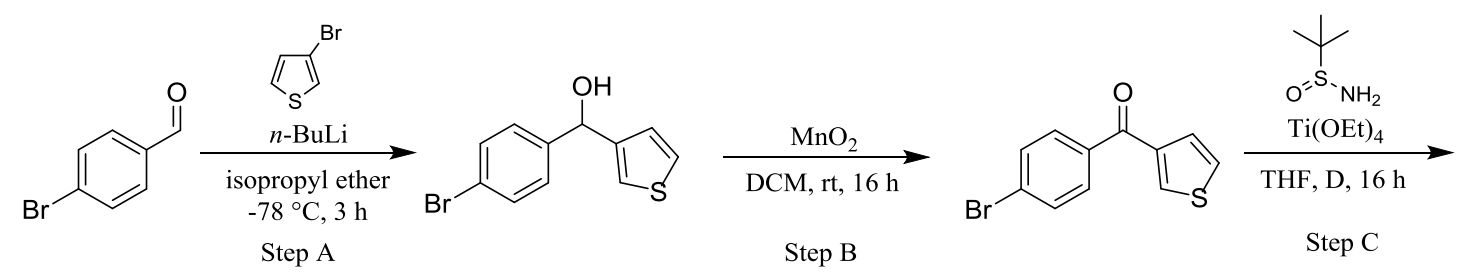

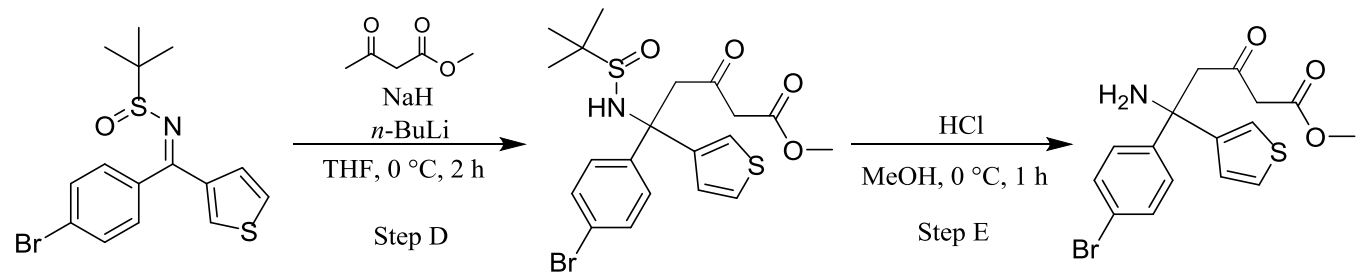

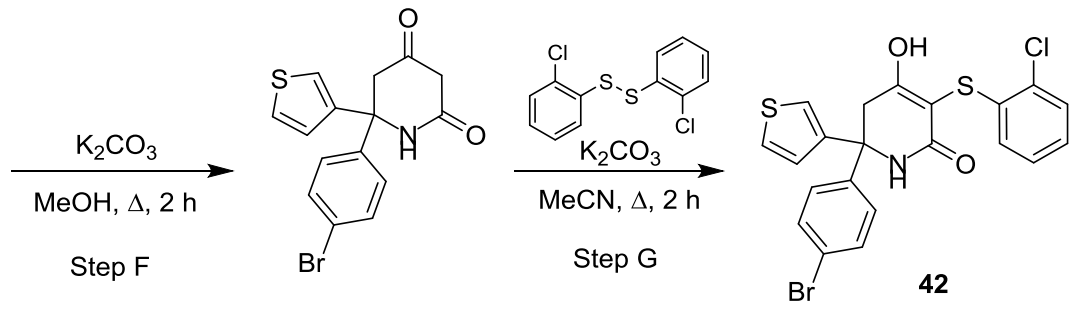

Step A: $n$-BuLi $(650 \mathrm{~mL}, 2.5 \mathrm{M}, 1.63 \mathrm{~mol})$ was slowly added to a solution of 3bromothiophene $(265 \mathrm{~g}, 1.63 \mathrm{~mol})$ in isopropyl ether $(3 \mathrm{~L})$ under $-78{ }^{\circ} \mathrm{C}$. After stirring at -78 ${ }^{\circ} \mathrm{C}$ for $30 \mathrm{~min}$, the reaction mixture was slowly treated with 4-bromobenzaldehyde $(250 \mathrm{~g}$, $1.35 \mathrm{~mol})$ in THF $(500 \mathrm{~mL})$ and stirred at $-78^{\circ} \mathrm{C}$ for 2 hours. The reaction mixture was quenched with sat. $\mathrm{NH}_{4} \mathrm{Cl}(1.5 \mathrm{~L})$, warmed to ambient temperature. The mixture was extracted with ethyl acetate $(800 \mathrm{~mL} \times 3)$. The organic layer was dried over $\mathrm{Na}_{2} \mathrm{SO}_{4}$ and concentrated. The crude product was purified by silica gel chromatography eluting with a gradient of $0 \%$ ethyl acetate/petroleum ether to $20 \%$ ethyl acetate/petroleum ether to afford (4-bromophenyl)(thiophen-3-yl)methanol (335 g, 92\%) as yellow oil. ${ }^{1} \mathrm{H}$ NMR (400 MHz, DMSO- $\left.d_{6}\right) \delta 7.51(\mathrm{~d}, J=8.4 \mathrm{~Hz}, 2 \mathrm{H}), 7.44(\mathrm{dd}, J=4.8 \mathrm{~Hz}, 2.8 \mathrm{~Hz}, 1 \mathrm{H}), 7.34(\mathrm{~d}, J=8.0 \mathrm{~Hz}$, $2 \mathrm{H}), 7.31(\mathrm{~d}, J=2.8 \mathrm{~Hz}, 1 \mathrm{H}), 6.98(\mathrm{~d}, J=4.4 \mathrm{~Hz}, 1 \mathrm{H}), 5.96(\mathrm{~d}, J=4.0 \mathrm{~Hz}, 1 \mathrm{H}), 5.75(\mathrm{~d}, J=$ $4.0 \mathrm{~Hz}, 1 \mathrm{H})$.

Step B: A mixture of (4-bromophenyl)(thiophen-3-yl)methanol (335 g, $1.25 \mathrm{~mol}), \mathrm{MnO}_{2}$ $(1083 \mathrm{~g}, 12.4 \mathrm{~mol})$ in $\mathrm{CH}_{2} \mathrm{Cl}_{2}(3.5 \mathrm{~L})$ was stirred at room temperature for 16 hours. The mixture was filtered over a short pad of silica gel. The filtrate was concentrated to afford (4bromophenyl)(thiophen-3-yl)methanone (320 g, 96\%) as a yellowish solid, which was used in the next step without further purification. ${ }^{1} \mathrm{H}$ NMR (400 MHz, DMSO- $\left.d_{6}\right) \delta 8.27(\mathrm{~d}, J=$ $1.6 \mathrm{~Hz}, 1 \mathrm{H}), 7.79-7.73(\mathrm{~m}, 5 \mathrm{H}), 7.54$ (d, $J=4.8 \mathrm{~Hz}, 1 \mathrm{H})$.

Step C: A mixture of (4-bromophenyl)(thiophen-3-yl)methanone (320 g, $1.20 \mathrm{~mol})$, $\mathrm{Ti}(\mathrm{OEt})_{4}(850 \mathrm{~g}, 3.73 \mathrm{~mol}), 2$-methylpropane-2-sulfinamide $(302 \mathrm{~g}, 2.50 \mathrm{~mol})$ and THF (3.5 
L) was heated at $80{ }^{\circ} \mathrm{C}$ for 16 hours. The suspension was allowed to cool to ambient temperature. The mixture was pour into ice water, filtered, washed with ethyl acetate. The filtrate was extracted with ethyl acetate (1.5 L x 2), dried over $\mathrm{Na}_{2} \mathrm{SO}_{4}$ and concentrated. The crude was purified by silica gel chromatography eluting with a gradient of $0 \%$ ethyl acetate/ petroleum ether to $30 \%$ ethyl acetate/ petroleum ether to afford $(E)-N-((4-$ bromophenyl)(thiophen-3-yl)methylene)-2-methylpropane-2- sulfinamide (316 g, 71\%) as a yellow solid. LCMS M/Z $(\mathrm{M}+\mathrm{H})=370$.

Step D: Methyl 3-oxobutanoate (194 g, $1.67 \mathrm{~mol})$ was added to a suspension of $\mathrm{NaH}$ $(67.0 \mathrm{~g}, 1.68 \mathrm{~mol})$ in THF $(3 \mathrm{~L})$ under $0{ }^{\circ} \mathrm{C}$. The reaction mixture was then slowly treated with $n-\mathrm{BuLi}(670 \mathrm{~mL}, 2.5 \mathrm{M}, 1.68 \mathrm{~mol})$ and stirred under $0{ }^{\circ} \mathrm{C}$ for $30 \mathrm{~min},(E)-\mathrm{N}-((4-$ bromophenyl) (thiophen-3-yl)methylene)-2-methylpropane-2-sulfinamide (310 g, $0.838 \mathrm{~mol})$ in THF $(500 \mathrm{~mL})$ was added to the mixture and stirred under $0{ }^{\circ} \mathrm{C}$ for another 2 hours. The reaction mixture was poured into ice-water and warmed to ambient temperature. The mixture was extracted with ethyl acetate $(800 \mathrm{~mL} \times 2)$. The organic layer was dried over $\mathrm{Na}_{2} \mathrm{SO}_{4}$ and concentrated to afford methyl 5-(4-bromophenyl)-5-(1,1-dimethylethylsulfinamido)-3-oxo-5(thiophen-3-yl) pentanoate (800 g, crude) as dark oil, which was used in the next step without further purification. LCMS M/Z $(\mathrm{M}+\mathrm{Na})=508$.

Step E: $\mathrm{HCl} / \mathrm{MeOH}(4 \mathrm{M}, 400 \mathrm{~mL})$ was slowly added to a solution of methyl 5-(4bromophenyl)-5-(1,1-dimethylethylsulfinamido)-3-oxo-5-(thiophen-3-yl)pentanoate (400 g, crude) in $\mathrm{MeOH}(3 \mathrm{~L})$ under $0{ }^{\circ} \mathrm{C}$. The mixture was stirred at $0{ }^{\circ} \mathrm{C}$ for 1 hour, and then slowly acidified to $\mathrm{pH} 7$ using $3 \mathrm{~N} \mathrm{NaOH}$ at $0{ }^{\circ} \mathrm{C}$. The solvent was removed under vacuum. The crude product was extracted with ethyl acetate (1 L x 2), dried over $\mathrm{Na}_{2} \mathrm{SO}_{4}$ and concentrated to afford methyl 5-amino-5-(4-bromophenyl)-3-oxo-5-(thiophen-3-yl)pentanoate (360 g, crude) as dark oil, which was used in the next step without further purification. LCMS M/Z $\left(\mathrm{M}-\mathrm{NH}_{2}\right)=365$.

Step F: Potassium carbonate $(310 \mathrm{~g}, 2.25 \mathrm{~mol})$ was added to a solution of methyl 5amino-5-(4-bromophenyl)-3-oxo-5-(thiophen-3-yl)pentanoate (360 g, crude) in $\mathrm{MeOH}$ (3.5 L). The mixture was heated at $80{ }^{\circ} \mathrm{C}$ for 2 hours. The suspension was allowed to cool to ambient temperature. The solvent was removed under vacuum, the crude product was dissolved in water $(2 \mathrm{~L})$, washed with ethyl acetate $(1 \mathrm{~L} \times 2)$. The aqueous layer was acidified to $\mathrm{pH} 4$ using $3 \mathrm{~N}$ aqueous hydrochloride and extracted with ethyl acetate $(1.5 \mathrm{Lx} 3)$. The organic layer was washed with brine, dried over $\mathrm{Na}_{2} \mathrm{SO}_{4}$ and concentrated to give 6-(4bromophenyl)-4-hydroxy-6-(thiophen-3-yl)-5,6-dihydropyridin-2(1H)-one (160 g, crude) as brown oil, which was used in the next step without further purification. LCMS M/Z $(M+H)=$ 350 .

Step G: Potassium carbonate (190 g, $1.38 \mathrm{~mol}$ ) and 1,2-bis(2-chlorophenyl)disulfane $(80.0 \mathrm{~g}, 0.279 \mathrm{mmol})$ was added to a solution of 6-(4-bromophenyl)-4-hydroxy-6-(thiophen3-yl)-5,6- dihydropyridin-2(1H)-one in $\mathrm{MeCN}(2 \mathrm{~L})$. The mixture was heated at $80{ }^{\circ} \mathrm{C}$ for 2 hours. The suspension was allowed to cool to ambient temperature. The solvent was removed under vacuum. The crude product was dissolved in water $(2 \mathrm{~L})$, and acidified to $\mathrm{pH} 4 \mathrm{using} 3$ $\mathrm{N} \mathrm{HCl}$. The aqueous layer was extracted with ethyl acetate $(1.5 \mathrm{~L} \times 2)$. The organic layer was 
washed with brine, dried over $\mathrm{Na}_{2} \mathrm{SO}_{4}$ and concentrated. The crude was purified by silica gel chromatography eluting with a gradient of $0 \%$ ethyl acetate/hexanes to $50 \%$ ethyl acetate/hexanes to afford 6-(4-bromophenyl)-3-((2-chlorophenyl)thio)-4-hydroxy-6(thiophen-3-yl)-5,6-dihydropyridin-2(1H)-one 42 (190 g, 84\%) as a white solid. LCMS M/Z $(\mathrm{M}+\mathrm{H})=492$.

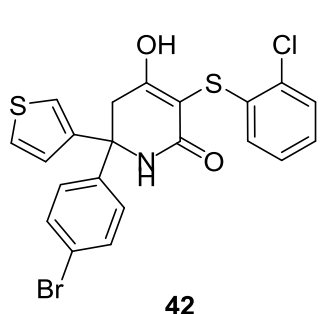

42

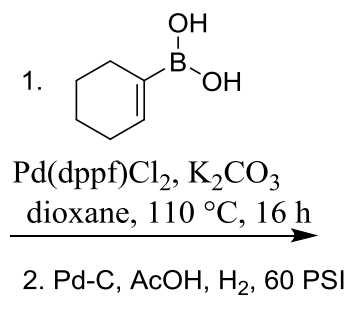

2. $\mathrm{Pd}-\mathrm{C}, \mathrm{AcOH}, \mathrm{H}_{2}, 60 \mathrm{PSI}$

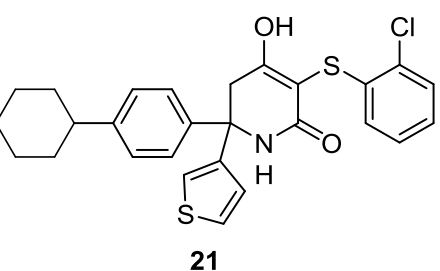

21

\section{3-(2-Chlorophenyl)sulfanyl-6-(4-cyclohexylphenyl)-6-(3-thienyl)piperidine-2,4-}

dione (21): To a solution of 6-(4-bromophenyl)-3-((2-chlorophenyl)thio)-6-(thiophen-3-yl) piperidine-2,4-dione $42(0.25 \mathrm{~g}, 0.5 \mathrm{mmol})$ in dioxane $(6 \mathrm{~mL})$ and water $(2 \mathrm{~mL})$ was added cyclohex-1-en-1-ylboronic acid (126 mg, $1 \mathrm{mmol}), \mathrm{Pd}(\mathrm{dppf}) \mathrm{Cl}_{2}(36 \mathrm{mg}, 0.05 \mathrm{mmol})$ and $\mathrm{K}_{2} \mathrm{CO}_{3}(0.27 \mathrm{~g}, 2 \mathrm{mmol})$. The reaction mixture was microwaved at $100{ }^{\circ} \mathrm{C}$ for 1 hour under nitrogen atmosphere. After cooling to room temperature, the reaction mixture was filtered through a short pad of silica gel. The filtrate was concentrated under vacuum and the crude residue was purified by preparative HPLC (formic acid) to afford 3-(2-chlorophenyl)sulfanyl6-[4-(cyclohexen-1-yl)phenyl]-6-(3-thienyl)piperidine-2,4-dione (11.7 mg, 5\% yield). ${ }^{1} \mathrm{H}$ NMR (400 MHz, DMSO-d $\left.{ }_{6}\right) \delta 8.47(\mathrm{~s}, 1 \mathrm{H}), 7.56-7.55(\mathrm{~m}, 1 \mathrm{H}), 7.54$ - 7.39(m, 2H), 7.32 $7.20(\mathrm{~m}, 3 \mathrm{H}), 7.27(\mathrm{~d}, J=8 \mathrm{~Hz}, 1 \mathrm{H}), 7.14(\mathrm{dd}, J=5.2,4.8 \mathrm{~Hz}, 1 \mathrm{H}), 6.93(\mathrm{dd}, J=7.6,4.8 \mathrm{~Hz}$, $1 \mathrm{H}), 6.15(\mathrm{~s}, 1 \mathrm{H}), 5.85(\mathrm{~d}, J=8.0 \mathrm{~Hz}, 1 \mathrm{H}), 3.39(\mathrm{~s}, 2 \mathrm{H}), 2.47(\mathrm{~s}, 2 \mathrm{H}), 2.33(\mathrm{~s}, 2 \mathrm{H}), 1.71$ - 1.68 $(\mathrm{m}, 2 \mathrm{H}), 1.58-1.56(\mathrm{~m}, 2 \mathrm{H})$. LCMS M+1 = 493.9.

To a solution of 3-(2-chlorophenyl)sulfanyl-6-[4-(cyclohexen-1-yl)phenyl]-6-(3thienyl)piperidine-2,4-dione $(0.8 \mathrm{~g}, 1.6 \mathrm{mmol})$ in acetic acid $(20 \mathrm{~mL})$ was added $\mathrm{Pd} / \mathrm{C}(0.1 \mathrm{~g})$. The reaction mixture was stirred at room temperature for 24 hours under hydrogen atmosphere (60 PSI). After relieving the pressure, the reaction mixture was filtered over Celite and the filtrate was concentrated under vacuum. The crude residue was purified by preparative HPLC (formic acid) to afford the product 21 (10 mg, 1.2\% yield) as white solid. ${ }^{1} \mathrm{H}$ NMR $(400 \mathrm{MHz}$, DMSO-d 6 ) $\delta 7.49(\mathrm{~s}, 1 \mathrm{H}), 7.35-7.32(\mathrm{~m}, 2 \mathrm{H}), 7.26-7.25(\mathrm{~m}, 4 \mathrm{H}), 7.19$ $(\mathrm{d}, J=8.0 \mathrm{~Hz}, 1 \mathrm{H}), 6.93(\mathrm{dd}, J=6.8,6.8 \mathrm{~Hz}, 1 \mathrm{H}), 6.72(\mathrm{dd}, J=6.8,6.8 \mathrm{~Hz}, 1 \mathrm{H}), 5.98(\mathrm{~d}, J=$ $6.8 \mathrm{~Hz}, 1 \mathrm{H}), 3.45$ (s, 2H), $1.96-1.74(\mathrm{~m}, 5 \mathrm{H}), 1.48-1.27$ (m, 5H). LCMS M+1 = 495.8. 


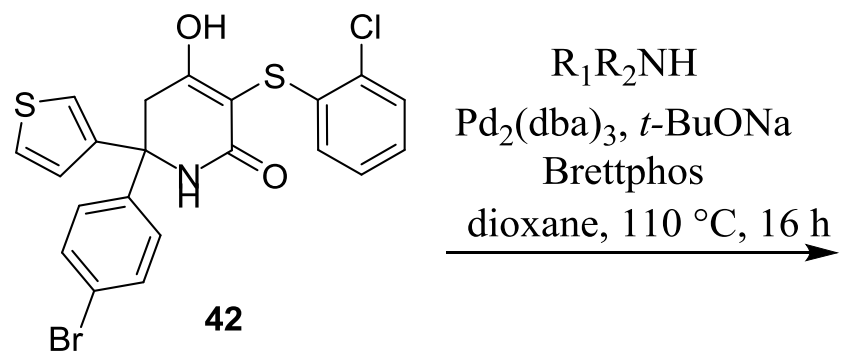<smiles>[R]N([R])c1ccc(C2(c3ccsc3)CC(O)=C(Sc3ccccc3Cl)C(=O)N2)cc1</smiles>

$24,29,31,33$

$$
\begin{gathered}
\mathrm{R}_{1} \mathrm{R}_{2} \mathrm{NH} \\
\mathrm{Pd}_{2}(\mathrm{dba})_{3}, \mathrm{Cs}_{2} \mathrm{CO}_{3} \\
\underset{\text { X-Phos, dioxane, } 100{ }^{\circ} \mathrm{C}, 12 \mathrm{~h}}{\longrightarrow}
\end{gathered}
$$<smiles>[R]N([R])c1ccc(C2(c3ccsc3)CC(O)=C(Sc3ccccc3Cl)C(=O)N2)cc1</smiles>

34-36

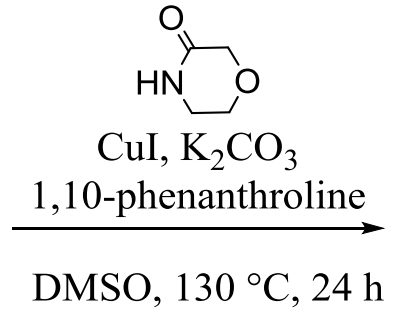<smiles>O=C1NC(c2ccc(N3CCOCC3=O)cc2)(c2ccsc2)CC(O)=C1Sc1ccccc1Cl</smiles>

(6R)-3-(2-Chlorophenoxy)-6-(4-morpholinophenyl) -6-(thiophen-3-yl)piperidine-2,4dione (29): To a solution of 6-(4-bromophenyl)-3-(2-chlorophenoxy)-6-(thiophen-3-yl) piperidine-2,4-dione $(600 \mathrm{mg}, 1.26 \mathrm{mmol})$ in dioxane $(10 \mathrm{~mL})$ was added morpholine (328 $\mathrm{mg}, 3.77 \mathrm{mmol})$, Brettphos (65 mg, 0.13mmol), $\mathrm{Pd}_{2}(\mathrm{dba})_{3}(64 \mathrm{mg}, 0.07 \mathrm{mmol})$ and t-BuONa (362 mg, $3.77 \mathrm{mmol}$ ). The solution was stirred for $8 \mathrm{~h}$ at $110 \mathrm{oC}$ under nitrogen. The solvent was removed under vacuum and the residue was purified by Prep-HPLC (FA) and chiral superfluid chromtography (stationary phase: Welko, $\mathrm{MeOH}$ w/ $0.1 \% \mathrm{NH}_{4} \mathrm{OH}$ ) to afford (6R)-3-(2-chlorophenoxy)-6-(4-morpholinophenyl)-6-(thiophen-3-yl)piperidine-2,4-dione (35 mg, 6\%) as white solid: ${ }^{1} \mathrm{H}$ NMR $\left(400 \mathrm{MHz}, \mathrm{DMSO}-\mathrm{d}_{6}\right) \delta 8.11(\mathrm{~s}, 1 \mathrm{H}), 7.54(\mathrm{dd}, \mathrm{J}=5.0$, $2.9 \mathrm{~Hz}, 1 \mathrm{H}), 7.35-7.22(\mathrm{~m}, 1 \mathrm{H}), 7.25(\mathrm{dd}, \mathrm{J}=8.2,5.5 \mathrm{~Hz}, 3 \mathrm{H}), 7.14(\mathrm{dd}, \mathrm{J}=5.1,1.4 \mathrm{~Hz}, 1 \mathrm{H})$, $6.93(\mathrm{t}, \mathrm{J}=10.1 \mathrm{~Hz}, 3 \mathrm{H}), 6.74(\mathrm{t}, \mathrm{J}=7.7 \mathrm{~Hz}, 1 \mathrm{H}), 5.95(\mathrm{~d}, \mathrm{~J}=8.0 \mathrm{~Hz}, 1 \mathrm{H}), 3.80-3.66(\mathrm{~m}$, $4 \mathrm{H}), 3.33(\mathrm{~s}, 2 \mathrm{H}), 3.16-3.05(\mathrm{~m}, 4 \mathrm{H}) ;{ }^{13} \mathrm{C}$ NMR (101 MHz, DMSO-d6) $\delta 173.52,166.20$, 149.94, 146.66, 137.45, 134.92, 128.87, 128.62, 127.29, 126.87, 126.75, 126.46, 125.00, $124.79,121.44,114.45,93.20,65.99,59.06,48.15,42.37$; HRMS $\left(\mathrm{ESI}^{+}\right)$calcd for $\mathrm{C}_{25} \mathrm{H}_{24} \mathrm{O}_{3} \mathrm{~N}_{2} \mathrm{ClS}_{2}(\mathrm{M}+\mathrm{H})^{+}$499.0911, found 499.0910.

Compounds 24, 31 and 33 were prepared using the conditions described for compound 29.

3-((2-Chlorophenyl)thio)-6-(4-(4-methoxypiperidin-1-yl)phenyl)-6-(thiophen-3yl)piperidine-2,4-dione (24): ${ }^{1} \mathrm{H}$ NMR $\left(400 \mathrm{MHz}, \mathrm{DMSO}_{6} \mathrm{~d}_{6}\right) \delta$ 7.60-7.55 (m, 5H), 7.37 (dd, $\mathrm{J}=4.2,4.2 \mathrm{~Hz}, 1 \mathrm{H}) 7.24(\mathrm{~d}, \mathrm{~J}=8.0 \mathrm{~Hz}, 1 \mathrm{H}), 7.17(\mathrm{~d}, \mathrm{~J}=4.0 \mathrm{~Hz}, 1 \mathrm{H}), 6.96(\mathrm{dd}, \mathrm{J}=8.0,8.0 \mathrm{~Hz}$, 
$1 \mathrm{H}), 6.10(\mathrm{~d}, \mathrm{~J}=8.0 \mathrm{~Hz}, 1 \mathrm{H}), 3.76-3.74(\mathrm{~m}, 2 \mathrm{H}), 3.57-3.55(\mathrm{~m}, 1 \mathrm{H}), 3.52-3.45(\mathrm{~m}, 4 \mathrm{H}), 3.42(\mathrm{~s}$, $3 \mathrm{H}), 2.24-2.06(\mathrm{~m}, 4 \mathrm{H})$.

3-((2-Chlorophenyl)thio)-6-(4-(2,6-dimethylmorpholino)phenyl)-6-(thiophen-3yl)piperidine-2,4-dione (31): ${ }^{1} \mathrm{H}$ NMR $\left(400 \mathrm{MHz}\right.$, Methanol-d $\left.\mathrm{d}_{4}\right) \delta 7.48(\mathrm{dd}, J=5.2,3.2 \mathrm{~Hz}$, $1 \mathrm{H}), 7.34-7.32(\mathrm{~m}, 2 \mathrm{H}), 7.31(\mathrm{~d}, J=3.2 \mathrm{~Hz}, 1 \mathrm{H}), 7.28(\mathrm{~d}, J=2.4 \mathrm{~Hz}, 1 \mathrm{H}), 7.27(\mathrm{~d}, J=2.4$ $\mathrm{Hz}, 1 \mathrm{H}), 6.99-6.97(\mathrm{~m}, 2 \mathrm{H}), 6.90(\mathrm{dd}, J=5.2,2.4 \mathrm{~Hz}, 1 \mathrm{H}), 6.73(\mathrm{dd}, J=5.2,2.4 \mathrm{~Hz}, 1 \mathrm{H})$, $6.06(\mathrm{dd}, J=8.0,1.2 \mathrm{~Hz}, 1 \mathrm{H}), 3.85-3.78(\mathrm{~m}, 2 \mathrm{H}), 3.36(\mathrm{~d}, J=16.0 \mathrm{~Hz}, 2 \mathrm{H}), 3.32(\mathrm{t}, J=5.2$ $\mathrm{Hz}, 2 \mathrm{H}), 3.38(\mathrm{t}, J=5.2 \mathrm{~Hz}, 2 \mathrm{H}), 1.38(\mathrm{~s}, 6 \mathrm{H})$.

3-((2-Chlorophenyl)thio)-6-(4-(2,2-dimethylmorpholino)phenyl)-6-(thiophen-3yl)piperidine-2,4-dione (33): ${ }^{1} \mathrm{H}$ NMR (400 MHz, Methanol-d4) $\delta 7.48(\mathrm{dd}, J=5.2 \mathrm{~Hz}, 5.2$ $\mathrm{Hz}, 1 \mathrm{H}), 7.33(\mathrm{~d}, J=8.8 \mathrm{~Hz}, 2 \mathrm{H}), 7.24(\mathrm{~d}, J=3.2 \mathrm{~Hz}, 1 \mathrm{H}), 7.18(\mathrm{~d}, J=8.8 \mathrm{~Hz} 1 \mathrm{H}), 7.15(\mathrm{~d}, J$ $=3.2 \mathrm{~Hz}, 1 \mathrm{H}), 7.14(\mathrm{~d}, J=8.8 \mathrm{~Hz}, 2 \mathrm{H}), 7.01(\mathrm{dd}, J=7.6 \mathrm{~Hz}, 7.6 \mathrm{~Hz}, 1 \mathrm{H}), 6.75(\mathrm{dd}, J=7.6 \mathrm{~Hz}$, $7.6 \mathrm{~Hz} 1 \mathrm{H}), 5.94(\mathrm{dd}, J=7.6 \mathrm{~Hz}, 7.6 \mathrm{~Hz} 1 \mathrm{H}), 3.88(\mathrm{t}, J=4.8 \mathrm{~Hz}, 2 \mathrm{H}), 3.40(\mathrm{~s}, 2 \mathrm{H}), 3.16(\mathrm{t}, J$ $=4.8 \mathrm{~Hz}, 2 \mathrm{H}), 3.03(\mathrm{~s}, 2 \mathrm{H}), 1.32(\mathrm{~s}, 6 \mathrm{H})$.

Compounds 34 - 36 were prepared as described for compound 29 using X-phos as ligand and cesium carbonate as base.

3-((2-Chlorophenyl)thio)-6-(4-(1,1-dioxidothiomorpholino)phenyl)-6-(thiophen-3yl)piperidine-2,4-dione (34): ${ }^{1} \mathrm{H}$ NMR (400 MHz, DMSO-d $\left.{ }_{6}\right) \delta 11.43(\mathrm{~s}, 1 \mathrm{H}), 8.39(\mathrm{~s}, 1 \mathrm{H})$, $7.57-7.51(\mathrm{~m}, 1 \mathrm{H}), 7.31-7.21(\mathrm{~m}, 4 \mathrm{H}), 7.14(\mathrm{~d}, \mathrm{~J}=4.0 \mathrm{~Hz}, 1 \mathrm{H}), 7.00(\mathrm{~d}, \mathrm{~J}=8.8 \mathrm{~Hz}, 2 \mathrm{H})$, 6.97 - $6.91(\mathrm{~m}, 1 \mathrm{H}), 6.74-6.67(\mathrm{~m}, 1 \mathrm{H}), 3.81-3.72(\mathrm{~m}, 4 \mathrm{H}), 3.37(\mathrm{~s}, 2 \mathrm{H}), 3.13-3.04(\mathrm{~m}$, $4 \mathrm{H})$.

6-(4-(2-Oxa-7-azaspiro[3.5]nonan-7-yl)phenyl)-3-((2-chlorophenyl)thio)-6(thiophen-3-yl)piperidine-2,4-dione (35): ${ }^{1} \mathrm{H}$ NMR (400 MHz, Methanol- $\left.\mathrm{d}_{4}\right) \delta 7.47(\mathrm{dd}$, $\mathrm{J}=5.2,3.2 \mathrm{~Hz}, 1 \mathrm{H}), 7.29-7.25(\mathrm{~m}, 3 \mathrm{H}), 7.19$ (d, J=8.0 Hz, $1 \mathrm{H}), 7.13(\mathrm{~d}, \mathrm{~J}=8.0 \mathrm{~Hz}, 1 \mathrm{H})$, 6.98(d, J=8.8 Hz, $2 \mathrm{H}), 6.90$ (dd, J = 8.0,8.0 Hz, $1 \mathrm{H}), 6.73$ (dd, J=8.0,8.0 Hz, $1 \mathrm{H}), 5.98$ (d, $\mathrm{J}=7.6 \mathrm{~Hz}, 1 \mathrm{H}), 4.48(\mathrm{~s}, 4 \mathrm{H}), 3.40(\mathrm{~s}, 2 \mathrm{H}), 3.14(\mathrm{t}, \mathrm{J}=5.6 \mathrm{~Hz}, 4 \mathrm{H}), 1.99$ (t, J=5.6 Hz, $4 \mathrm{H})$.

1-(4-(5-((2-Chlorophenyl)thio)-4,6-dioxo-2-(thiophen-3-yl)piperidin-2yl)phenyl)piperidine-4-carbonitrile (36): ${ }^{1} \mathrm{H}$ NMR $\left(400 \mathrm{MHz}\right.$, Methanol- $\left.\mathrm{d}_{4}\right) \delta 7.43(\mathrm{dd}$, $\mathrm{J}=7.6,2.4 \mathrm{~Hz}, 1 \mathrm{H}), 7.30$ (d, J=8.8 Hz, 2H), 7.23 (d, J=3.2 Hz, 1H), 7.13-7.10 (m, 2H), 6.97 $(\mathrm{d}, \mathrm{J}=8.8 \mathrm{~Hz}, 2 \mathrm{H}), 6.82(\mathrm{dd}, \mathrm{J}=7.6,7.6 \mathrm{~Hz}, 1 \mathrm{H}), 6.70(\mathrm{dd}, \mathrm{J}=7.6,7.6 \mathrm{~Hz}, 1 \mathrm{H}), 6.05(\mathrm{~d}, \mathrm{~J}=7.6$ $\mathrm{Hz}, 1 \mathrm{H}), 3.42$ (t, J=5.2 Hz, 2H), $3.28(\mathrm{~s}, 2 \mathrm{H}), 3.09$ (t, J=5.2 Hz, 2H), 3.01-2.94 (m, 1H), 2.03 (t, J=5.2 Hz, 2H), 1.92 (t, J=5.2 Hz, 2H).

3-((2-Chlorophenyl)thio)-6-(4-(3-oxomorpholino)phenyl)-6-(thiophen-3yl)piperidine-2,4-dione (32):To a solution of 6-(4-bromophenyl)-3-((2-chlorophenyl)thio)-6(thiophen-3-yl)piperidine-2,4-dione $(300 \mathrm{mg}, 0.6 \mathrm{mmol})$ in DMSO $(5 \mathrm{~mL})$ was added copper(I) iodide (23 mg, $0.12 \mathrm{mmol}$ ), 1,10-phenanthroline (10 mg, $0.06 \mathrm{mmol}$ ), $\mathrm{K}_{2} \mathrm{CO}_{3}$ (168 $\mathrm{mg}, 1.2 \mathrm{mmol})$ and 4-iodo- morpholin-3-one (123 $\mathrm{mg}, 1.2 \mathrm{mmol})$. The reaction mixture was heated to $130{ }^{\circ} \mathrm{C}$ for $24 \mathrm{~h}$ under a nitrogen atmosphere. After cooling to room temperature, the mixture was filtered and concentrated in vacuo. The crude residue was purified by reverse 
phase chromatography (formic acid) to afford 3-((2-chlorophenyl)thio)-6-(4-(3-

oxomorpholino)phenyl)-6-(thiophen-3-yl)piperidine-2,4-dione (56 mg, 18\%) as a white solid. ${ }^{1} \mathrm{H}$ NMR (400 MHz, $\left.\mathrm{CD}_{3} \mathrm{OD}\right) \delta 7.57$ - $7.52(\mathrm{~m}, 3 \mathrm{H}), 7.46$ - $7.41(\mathrm{~m}, 2 \mathrm{H}), 7.37(\mathrm{~s}, 1 \mathrm{H}), 7.26$ $7.19(\mathrm{~m}, 2 \mathrm{H}), 6.98-6.93(\mathrm{~m}, 1 \mathrm{H}), 6.91-6.84(\mathrm{~m}, 1 \mathrm{H}), 6.10(\mathrm{~d}, J=7.6 \mathrm{~Hz}, 1 \mathrm{H}), 4.31(\mathrm{~s}, 2 \mathrm{H})$, $4.07(\mathrm{t}, J=4.8 \mathrm{~Hz}, 1 \mathrm{H}), 3.82(\mathrm{t}, J=4.8 \mathrm{~Hz}, 1 \mathrm{H}), 3.52(\mathrm{~s}, 2 \mathrm{H})$.

Small molecule X-ray crystallography. X-ray quality crystals were grown from a saturated acetonitrile solution with the addition of 1 equivalent of tetramethylammonium hydroxide ( $25 \%$ solution in $\mathrm{MeOH}$ ) followed by the slow vapor diffusion of diisopropyl ether to deposit the crystal diffracted. A colorless prism $0.050 \times 0.030 \times 0.030 \mathrm{~mm}$ in size was mounted on a Cryoloop with Paratone oil. Data were collected in a nitrogen gas stream at $100^{\circ} \mathrm{K}$ using phi and omega scans. Crystal-to-detector distance was $40 \mathrm{~mm}$ and exposure time was 10 seconds per frame using a scan width of $1.0^{\circ}$. Data collection was $99.8 \%$ complete to $25.000^{\circ}$ in $\square$. A total of 55134 reflections were collected covering the indices, $-10<=h<=10,-21<=k<=21$, $21<=l<=22.5481$ reflections were found to be symmetry independent, with an $\mathrm{R}_{\text {int }}$ of 0.0793 . Indexing and unit cell refinement indicated a primitive, orthorhombic lattice. The space group was found to be $\mathrm{P} 2{ }_{1} 2{ }_{1} 2_{1}$ (No. 19). The data were integrated using the Bruker SAINT software program and scaled using the SADABS software program. Solution by iterative methods (SHELXT-2014) produced a complete heavy-atom phasing model consistent with the proposed structure All non-hydrogen atoms were refined anisotropically by full-matrix leastsquares (SHELXL-2014). All hydrogen atoms were placed using a riding model. Their positions were constrained relative to their parent atom using the appropriate HFIX command in SHELXL-2014. Absolute stereochemistry was unambiguously determined to be $R$ at $\mathrm{C} 1$. 


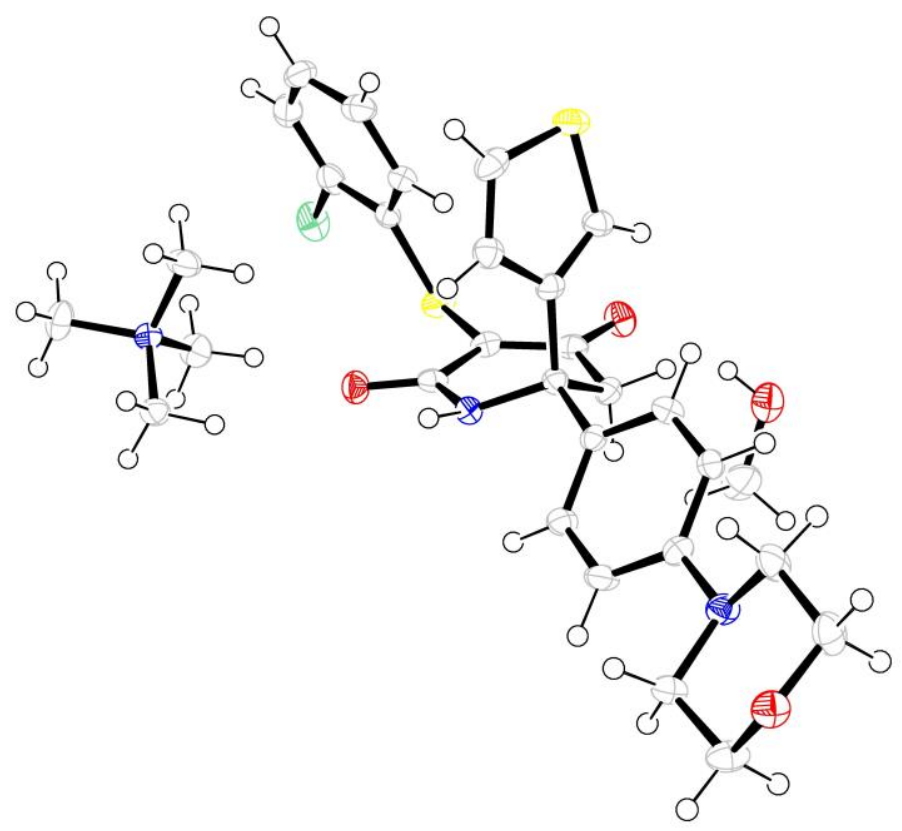

Figure S4. Small molecule crystal structure of compound 29.

Table S2. X-ray Structure Determination Data and Statistics

PDB code

Data collection/reduction

X-ray source

Wavelength $(\AA)$

Resolution range $(\AA)$

Space group

Unit cell edges $(\AA)$

Unit cell angles $\left(^{\circ}\right)$

Total reflections

Unique reflections

Multiplicity

Completeness (\%)

Mean I/ $\sigma(\mathrm{I})$
Compound 9/LDHA

5IXS

SSRL 11-1

0.9795

$37.8-2.05(2.128-2.05)$

$\mathrm{P} 2{ }_{1}$

74.6281 .23104 .80

90, 97.56, 90

265849

764670

3.5 (3.3)

98.2

$16.6(2.1)$
Compound 30/LDHA

5IXY

SSRF 17U

0.9791

33.1-3.0 (3.11 - 3.0)

$\mathrm{P} 2{ }_{1}$

78.4580 .70102 .24

90, 97.90, 90

75308

22595

3.1(3.3)

95.2

10.4(3.4) 


$\begin{array}{lll}\text { Wilson B-factor }\left(\AA^{2}\right) & 32.7 & 49.2 \\ \text { R-symm } & 0.058(0.599) & 0.103(0.402) \\ \text { Refinement } & & \\ \text { Reflections used for R-free } & 1571 & 506 \\ \text { R-work } & 0.174 & 0.236 \\ \text { R-free } & 0.221 & 0.279 \\ \text { Number of non-H atoms } & 11112 & 10593 \\ \text { macromolecules } & 10190 & 10250 \\ \text { ligands } & 353 & 343 \\ \text { water } & 569 & 0 \\ \text { Protein residues } & 1305 & 1321 \\ \text { RMS(bonds) }(\AA) & 0.007 & 0.008 \\ \text { RMS(angles) }\left({ }^{\circ}\right) & 1.4 & 1.1 \\ \text { Ramachandran favored }(\%) & 97 & 96 \\ \text { Average B-factor }\left(\AA^{2}\right) & 38.4 & 55.38 \\ \text { macromolecules } & 38.34 & 55.34 \\ \text { ligands } & 43.6 & 56.35 \\ \text { solvent } & 35.8 & -\end{array}$

